\title{
Intelligent Baby Behavior Monitoring using Embedded Vision in IoT for Smart Healthcare Centers
}

\author{
Tanveer Hussain ${ }^{a}$, Khan Muhammad ${ }^{b}$, Salman Khan', Amin Ullah, Mi Young Lee, \\ Sung Wook Baik ${ }^{\text {d,* }}$ \\ Intelligent Media Laboratory, Digital Contents Research Institute, Sejong University, Seoul 143-147, Republic of Korea \\ a. tanveerkhattak3797@gmail.com , b. khan.muhammad.icp@gmail.com , c. salmank3797@gmail.com, d.sbaik@sejong.ac.kr \\ *Corresponding author
}

How to cite this paper: Tanveer Hussain; Khan Muhammad; Salman Khan; Amin Ullah; Mi Young Lee; Sung Wook Baik (2019) Intelligent Baby Behavior Monitoring using Embedded Vision in IoT for Smart Healthcare Centers. Journal of Artificial Intelligence and Systems, 1, 110-124. https://doi.org/10.33969/AIS.2019.11007

Received: September 10, 2019

Accepted: October 21, 2019

Published: November 5, 2019

Copyright ( 2019 by author(s) and Institute of Electronics and Computer. This work is licensed under the Creative Commons Attribution International License (CC BY 4.0). http://creativecommons.org/licenses/by/4.0/ cc) (i) Open Access

\begin{abstract}
Mainstream Internet of Things (IoT) techniques for smart homes focus on appliances and surveillance in smart cities. Most of the researchers utilize vision sensors in IoT environment targeting only adult users for various applications such as abnormal activity recognition. This paper introduces a new paradigm in vision sensor IoT technologies by analyzing the behavior of baby through an intelligent multimodal system. Traditional wearable sensors such as heartbeat if attached to any body part of the baby make him uncomfortable and also some babies are paranoid toward sensors. Our vision based baby monitoring framework employs one of the process improvement techniques known as control charts to analyze the baby behavior. We construct control chart in a specific interval for real-time frames generated by Raspberry Pi (RPi) with attached vision sensor. Baby motion is represented through points on control chart, if it exceeds upper control limit (UCL) or falls from lower control limits (LCL), it indicates abnormal behavior of the baby. Whenever such a behavior is encountered, a signal is transmitted to the interconnected devices in IoT as an alert to baby care takers in smart health care centers. Our proposed framework is adaptable, a single RPi can be used to monitor a baby in home or a network of RPi's for an IoT in a children nursery for multiple babies monitoring. Performance evaluation on our own created dataset indicates the better accuracy and efficiency of our proposed framework.
\end{abstract}

\section{Keywords}

IoT, Smart Health Care, Nurseries Monitoring, Smart Hospitals, Image Processing, Resource Constrained Devices, Control Charts

\section{Introduction}

The recent advancements in IoT technology for various applications [1, 2] overwhelms the traditional sensing of surrounding environments. It is emerging very rapidly and becoming a major source for exchange of communication among different sensors including vision sensors over smart devices such as smartphones etc. These sensors are 
widely used in building smart homes [3] and cities for various services [4, 5], secure surveillance [6], and industries [7-10]. Among these technologies, many researchers are enthusiastic to make homes smarter through various IoT devices ranging from small utility items such as tooth brushes that can smartly monitor your brushing activities to smarter home surveillance. The main focus of researchers working in IoT domain for smart homes is limited to home appliances such as smart refrigerators, energy and lighting system, plugs/outlets, thermostats, and door locks. There are some research contributions focusing on assistance of elderly people for monitoring such as fall detection and nocturia monitoring systems [11, 12]. But the surveillance cameras of smart homes till date are used for security which limits the use of vision sensors in smart homes for monitoring only, thereby recognizing different actions and activities performed by adults. However, the vision sensors in smart homes can be used for many other applications including smart and intelligent monitoring of babies. Such applications of vision sensors can possibly be applied in smart hospitals and nurseries, assisting humans by reducing their efforts.

A simple baby monitoring system consists of different modules which monitor behavior and activities of babies and informs the care takers of them about abnormal or uncertain behavior through a text message, email or even displaying a message on the screen. In past, human families have instincts to protect their offspring form certain risks and dangers. However, in this contemporary era, for most of the working parents it is hard to look after their young born all the time. Taking advantage of this modern technological era, parents have adopted the new ways $[13,14]$ to look after their kids. Parents are now curious about new automatic techniques that can provide intelligent look after for their babies, thereby giving them greater chances of doing house chores and office work.

Of course there exists some other options for parents such as hiring a foster parent to look after babies and care centers, but it may not be feasible for them due to safety, surety, and their heavy expenses. On this behalf, researchers have contributed and designed several baby monitoring systems which can take care of the young born babies in the absence of their parents. Aslam et al. [15] presented a system which consists of RPi as processing unit, a microphone to detect baby cry, a motion detector for detecting motion of the baby, RPi camera for video streaming and display for video output. This system only activates when motion sensor detects motion of the baby or whenever microphone receives baby cry sound signals, alarm is set to on and camera starts streaming. A similar system designed by [16] adopts the same principle and when the baby start crying, this system start swinging the cradle until the baby stops crying. A study conducted by Madhuri et al. [14] utilizes the smartphone to monitor the baby. This system informs the parents when the baby does not stop crying in a specific duration of time. Megha et al. [17] used Arduino microcontroller and temperature sensors to control and adjust the temperature for the baby inside the incubator. Chaoyuan et al. [18] used deep neural network with Gaussian Mixture and Hidden Markov model to identify cry and to monitor 
the heat rate of the baby.

A deep analysis of the existing literature reveals that the monitoring systems proposed for babies consumes energy by implementing different sensors such as audio and vision sensors along with processing units. The existing systems are not adaptable and functional only with a large number of hardware requirements. Further, these systems do not take advantage of various smart devices in IoT. Some of the techniques rely on implementing hardware near or over body parts of the baby that proves to be problematic in some cases. Last but not the least, vision based existing baby monitoring systems process the baby video frame by frame, yielding false alarm generation as a result of frames level decision. Therefore, a complete, adaptable, energy efficient, easily implementable, and user friendly baby monitoring system is required that can intelligently monetarize the baby through vision sensor. A system that is intelligent enough to recognize an abnormal behavior of the baby and has instant alert notification module is the demand from parents nowadays. Thus, considering the limitations of the existing systems we pose a new framework in IoT for intelligent baby behavior monitoring. Our contributions are summarized as follows:

1) The techniques applied via emerging trends of IoT are limited to home appliances, lighting systems, and security cameras etc. Majority of these employed techniques ignore the special use of IoT sensors, helping parents in smart homes and assisting babies care takers in nurseries and smart health care centers. In this framework, we apply intelligent image processing techniques on frames acquired from smart vision sensor that is connected to the wireless sensor network (WSN) to analyze the behavior of the baby. Thus, under the umbrella of our novel framework there is no need for parents in smart homes or nurses in hospitals to monitor babies manually.

2) There are many problems of motion computation with frames level processing and decision making. For instance, it has higher probability of false alert/alarm generation if we produce alarm on the basis of motion between two frames. Thus, we introduce an intelligent framework that is able to generate alarm using control charts after analyzing an interval of video frame. For example, in a case if the baby is uncomfortable or not breathing for a short period of time continuously our system will produce alarm. So, we make contribution to IoT domain for smart healthcare centers by making the process intelligent and reducing false alarms generation.

3) The literature of IoT for smart homes, healthcare centers, and nurseries lacks benchmark datasets for performance evaluation of baby monitoring systems. In this paper, we created our own videos dataset captured for both day and night scenarios. In dark situations we used night vision camera to capture baby from different heights and poses so that our dataset is not applicable only for daylight. This dataset with the ground truth will be made publicly available for benchmarking to help healthcare experts and scientists for further research in this field.

4) The IoT environment consists of various sensors and devices that can be utilized for different purposes. In order to make our framework adaptable in every IoT 
scenario we also made experiments on a personal computer to not limit our framework only to the embedded devices. Thus, in IoT environment it is not mandatory for our framework that the vision sensor should be attached to the RPi for processing, rather it can be viewed live over smart displays and processed over smart computers and even smartphone devices that are easily accessible in daily routine.

The remainder of the paper is divided into three sections. Section II explains the working of our proposed baby monitoring framework. Section III provides detail about the experimental evaluation and visualizes the sample results of our proposed framework. Last section of the article concludes our framework and highlights the limitations of the current system and explains the future work.

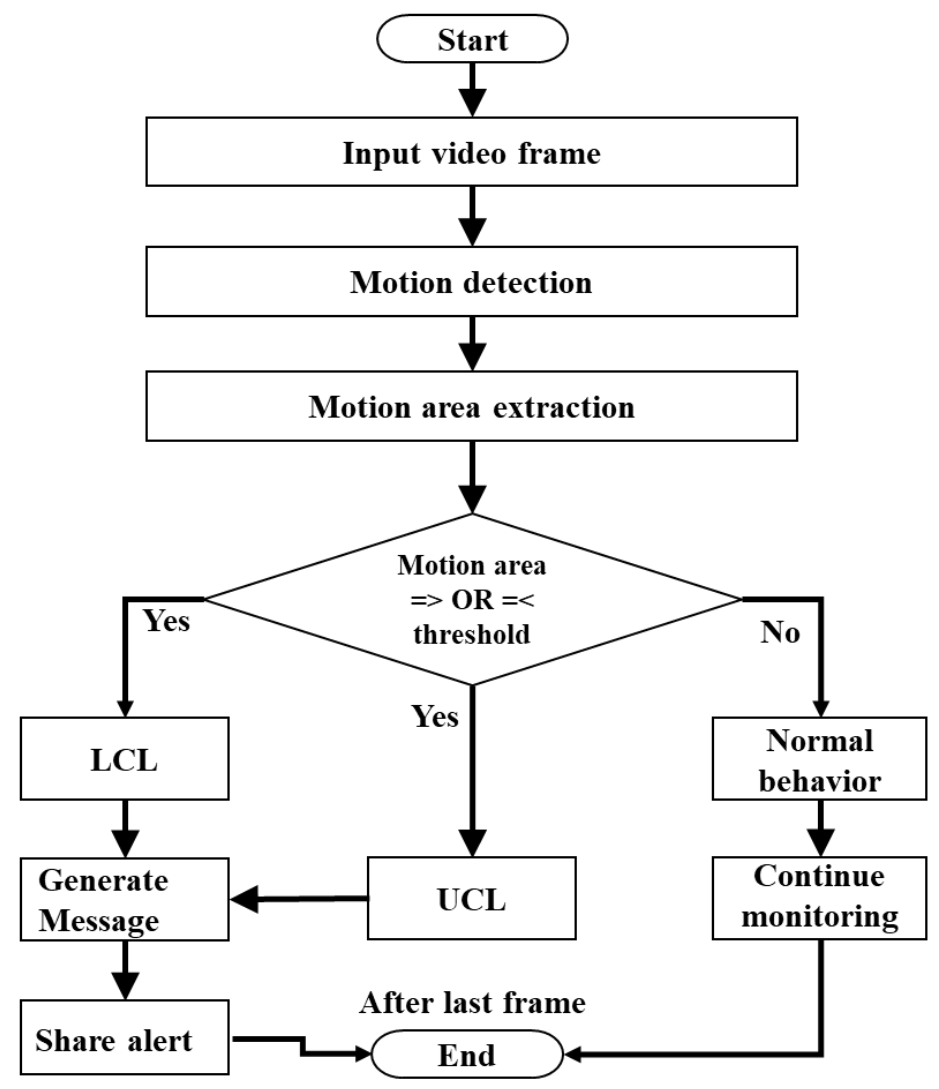

Figure 1. Process flow of overall proposed baby monitoring system

\section{Proposed Baby Monitoring System}

This section describes detail working of our proposed baby monitoring framework through control charts for smart homes and nurseries. An input in the form of video frames from the RPi with attached camera fixed over the baby bed is acquired. The video frames are passed to the motion detection mechanism where two different methods are applied individually and their results are integrated to achieve better accuracy. It outputs a binary image where the white pixels represent motion of the baby. This binary image is computed by control chart construction mechanism to draw a point on the chart. The 
control chart constructed for a whole interval (currently 5 frames per interval) is checked for abnormal behavior. If majority of the points crosses the upper or lower boundary in a single interval, it is marked as abnormal and alert is generated. The generated alert is transmitted to the guardians or parents for quick actions along with options to view the current state of the baby through live streaming. Process flow of overall system is given in Fig. 1 and the steps are visualized in Fig. 2.

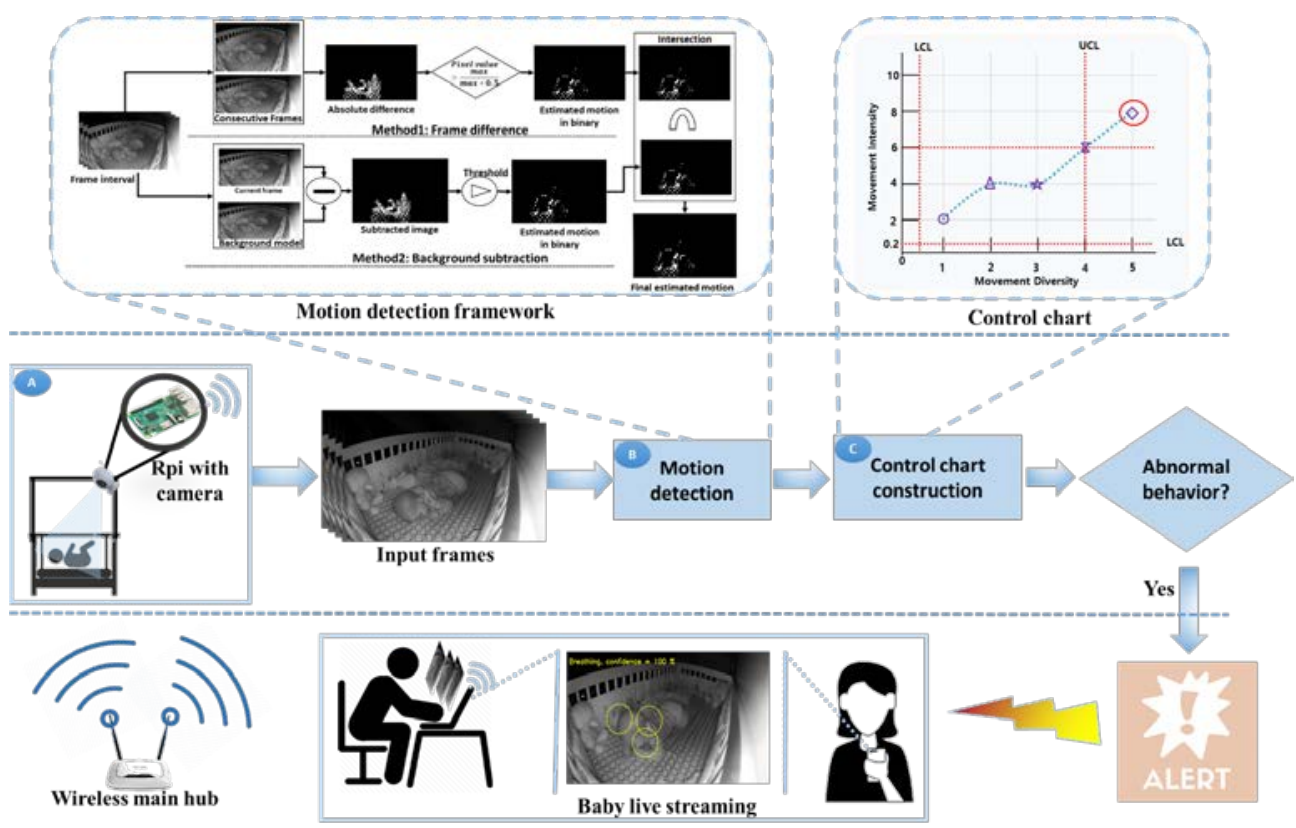

Figure 2. Proposed baby behaviour monitoring framework. (a): Input is acquired from smart camera attached to RPi, (b): motion computation where two different algorithms are executed to compute motion of baby, and (c): control chart construction from the detected motion, abnormal breathing behaviour detection on the basis of one interval of control chart, and alert transmission in case of any abnormal breathing or motion behaviour.

\subsection{Hardware Setup and Input Acquisition}

The hardware setup contains an RPi with a camera that is fixed over the bed of the baby. RPi has wireless sensor that is connected to the wireless network in smart home. The similar setup can be used in nurseries where a network of RPi's captures video data from a number of babies. The overall processing is done over the RPi and the camera attached to the RPi can possibly be a night vision camera working in both light and dark scenarios, as majority of the babies are used to sleep in dark. There is no restriction of using RPi with an attached camera, we have also made experiments with a setup where camera gives live coverage to a local computer, both connected to the same wireless network. Thus, the whole processing for baby monitoring is then performed over that local computer working together with the smart camera in an IoT environment. We acquire frames from the camera and apply two different motion detection algorithms to compute 
motion so as to detect every slight motion of baby. Working of motion detection algorithms is explained in the next section.

\subsection{Motion Detection}

Motion detection mechanism is based on two algorithms that are feasible to be implemented on any resource constrained device. Motion is computed for video frames by passing them to the frame difference and background subtraction algorithm. The overall flow of baby motion detection is shown in Fig. 3 and explained in coming sections.

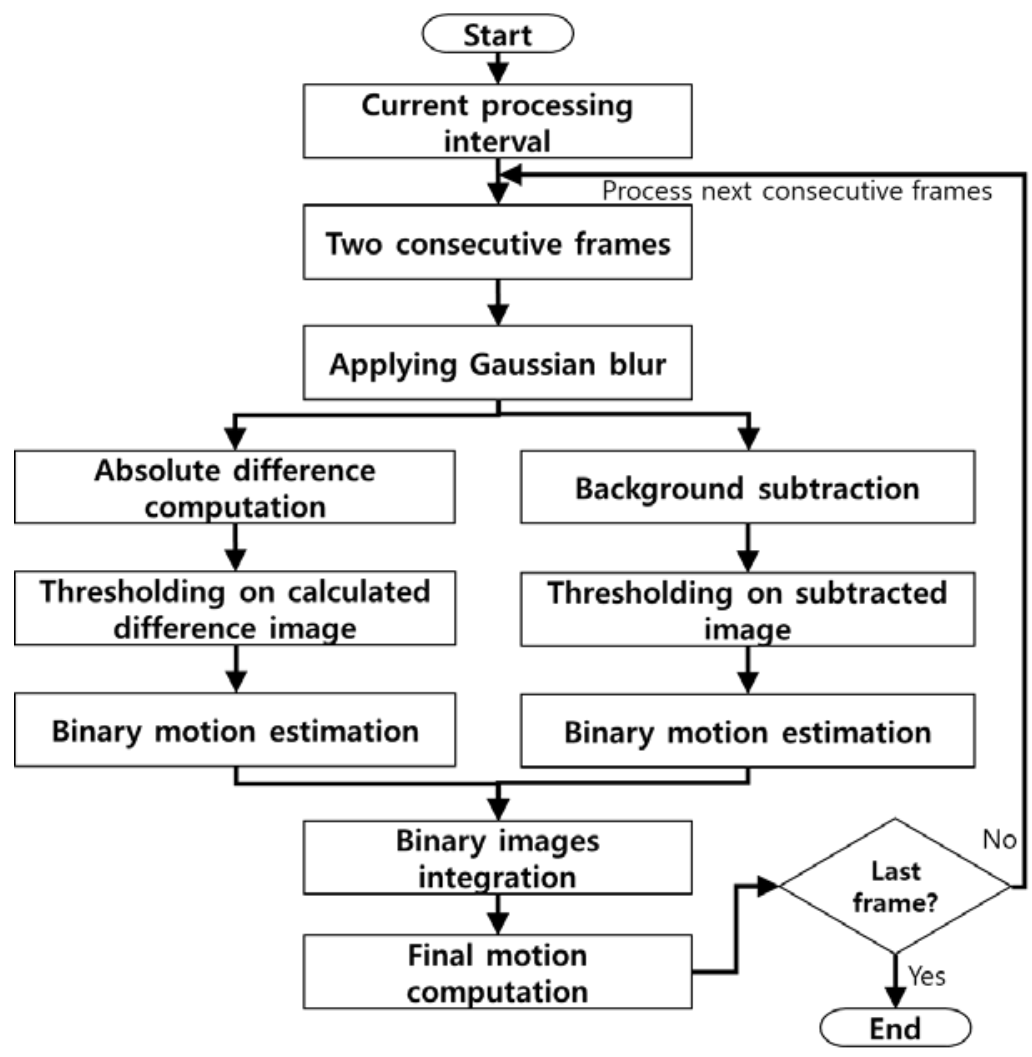

Figure 3. Flow of the proposed motion detection algorithm. This algorithm inputs two successive frames (i, $\mathrm{i}+1)$ form the first module and applies frame differencing and background subtraction algorithm. The outputs from these two algorithms are acquired in binary format where the two different binary images are integrated together by taking bitwise AND logical operation of these two images. The integrated binary image indicates the motion of the baby where it may be the motion of any body part of the baby or just breathing. The motion area is compared with a certain threshold which decides whether the baby is breathing or not and finally next two frames from the live video are acquired to loop back the same process.

\subsubsection{Frame difference method}

Frame difference based motion detection is one of the simplest and precise techniques to 
detect even a smaller change in pixels. First step of this technique involves capturing two consecutive frames $\left(f_{i}\right.$ and $\left.f_{i+1}\right)$ acquired from the baby live video. Second step involves some preprocessing of the input frames such as conversion into grayscale and applying Gaussian blur to remove noise. Next step is to calculate the pixel wise absolute difference between these two successive frames and store the difference image given as $d_{\text {image }}=1$ $f_{i+1}-f_{i} \mid$. For detailed analysis of the obtained difference image, it is converted into binary format. In case of no movement the binary image is black having all zero values and in the case of motion, the moving object in the binary image is illuminated with white color as shown graphically in Fig. 4 (b).

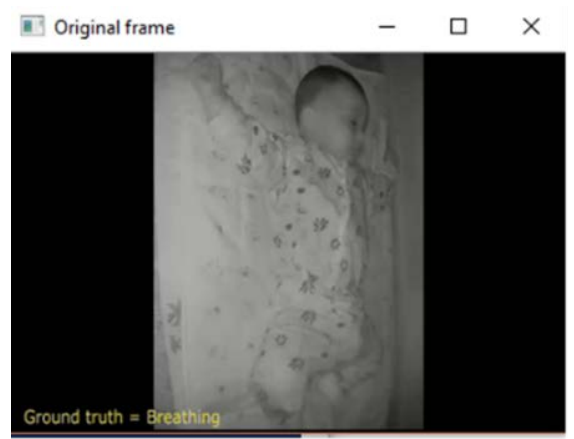

(a)

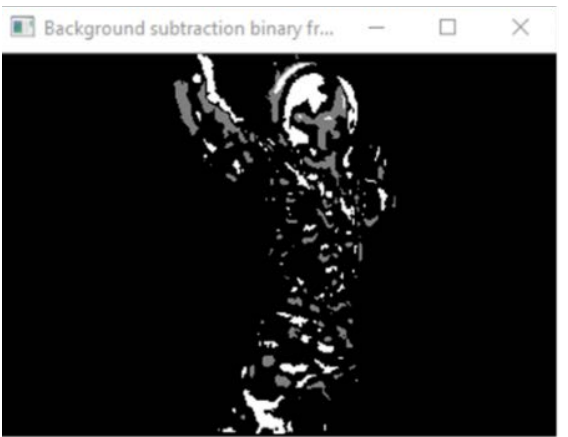

(c)

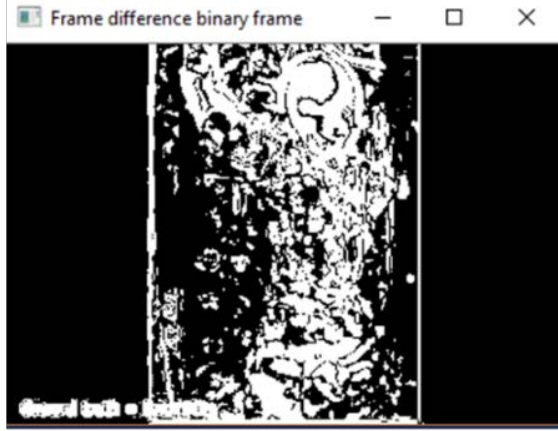

(b)

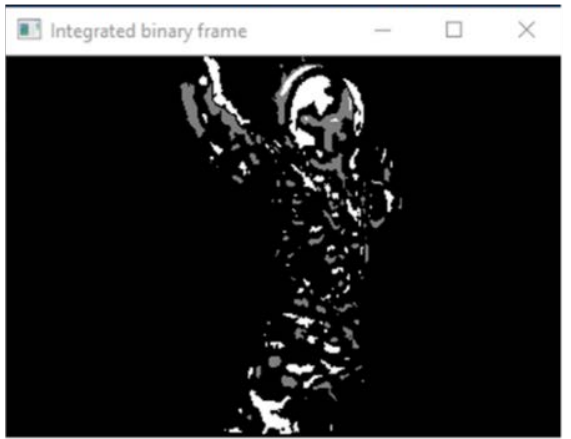

(d)

Figure 4. Sample results of baby sleeping video at night scenario through night vision camera. (a) Input frame with ground truth as breathing at the left bottom corner, (b) binary image formed from the frame difference method, (c) binary image of background subtraction model, and (d) final integrated binary frame through bitwise AND logical operation. This scenario contains static images so it is hard to observe motion from the input frame, but it can be clearly observed from the binary frames.

\subsubsection{Background subtraction method}

Background subtraction is a commonly used algorithm for motion detection problem in various computer vision applications. We used the background subtraction algorithm based on [19, 20]. In these papers, the authors utilized traditional pixel-level approach and recursive equations to develop an efficient adaptive algorithm via Gaussian mixture probability density. This algorithm is also known as "Gaussian Mixture-based Background/Foreground Segmentation”. It selects a suitable number of Gaussian 
distribution for each pixel where some other existing background subtraction algorithms fail to do so. Thus, through this concept it also provides better adaptability as compared to other background subtraction algorithms to varying scenes because of light changes and noise etc. The better results of background subtraction algorithm can be visualized in Fig. 4 (c).

\subsubsection{Integration of motion detection mechanisms}

The resultant frame in binary format is obtained from both motion computation algorithms independently and processed further to obtain the final binary frame. These two binary frames are integrated together through logical AND operation so as to get the common points of both the binary frames that are used by the control chart construction module defined in the subsequent sections.

\subsection{Breathing Behavior Analysis}

The last step of our proposed framework is behavior analysis of baby and transmission of an alert to the guardians in case of any abnormal motion or no motion. The breathing behavior of baby is analyzed via the concept of control charts in this paper. Control charts are graphs majorly used in process improvement techniques to study the change of different processes over time. There are two important concepts: UCL and LCL in control charts where UCL refers to very intense motion and LCL refers to no motion at all in our scenario. Crossing any of these limits for a specific number of frames in a single interval yields an alert transmission in the IoT network. The motion of the baby is sometime intense for only one part of the body or sometimes it is severe for many body parts. Diversity of motion is represented via number of circles around the motion areas. Intensity of motion is indicated through the size of circle drawn around the motion areas such that big circle shows high-level of motion and vice versa. These concepts are further elaborated in next sections.

\subsection{Control charts construction}

Control charts construction for a specific interval indicates the baby breathing behavior for that particular interval where it can be observed as normal or abnormal. Deciding on the basis of control chart of two frames is a biased decision as the motion or being static is very short between intervals of two frames. Therefore, we consider drawing control charts after an interval of five frames but it is not limited to this threshold and can be changed in accord to one's own requirements. Intensity of motion is plotted on Y-axis against diversity on $\mathrm{X}$-axis. The total area of the integrated binary image after motion computation is used to plot the $\mathrm{X}$-, $\mathrm{Y}$-axis values. The numbers of motion areas (white pixels) inside the binary image are plotted on $\mathrm{X}$-axis and the area of those white pixels is plotted on Y-axis. An important point to be noted that many circles (motion areas) are hard to identify for plotting through control chart as we do not know which circle should be plotted. Currently we do not apply any special mechanism but only select the circle with maximum area for plotting it on Y-axis. Control charts for a number of intervals of 
our own created dataset video are shown in Fig. 5.

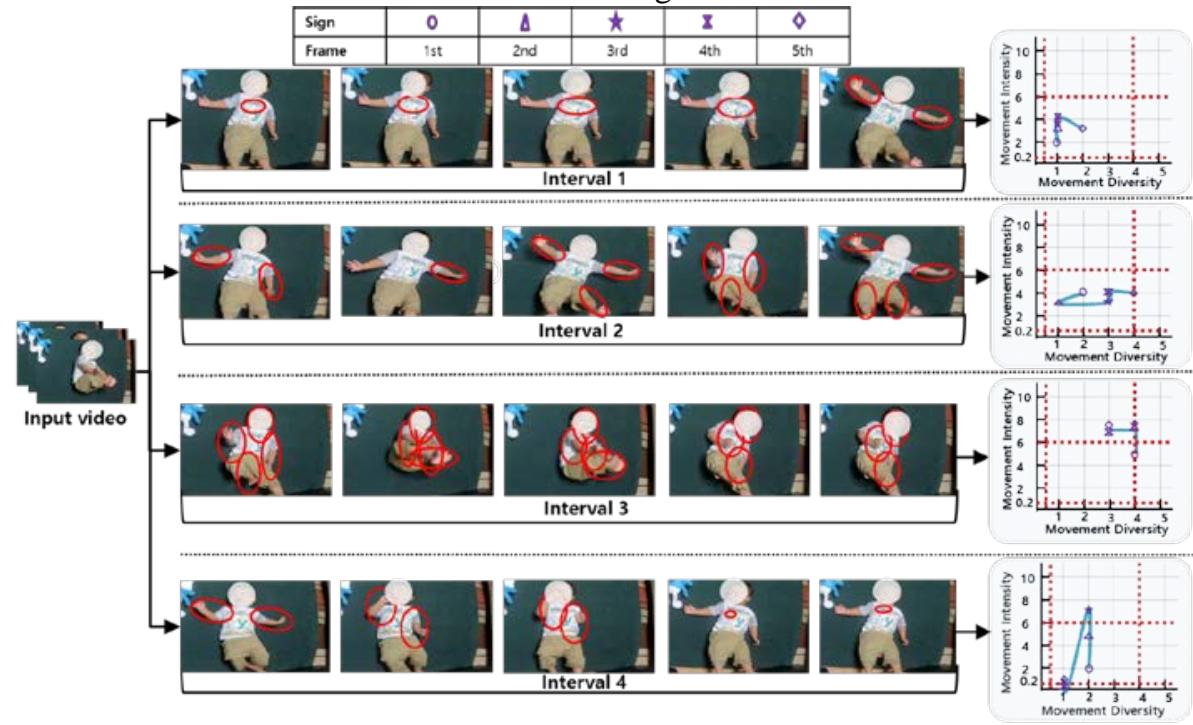

Figure 5. Sample frames of our own created baby video dataset. The first and second interval is normal as the threshold of more than two frames for being abnormal is not crossed whereas in interval 3 this limit is reached so it is abnormal interval. Similarly, in fourth interval majority frames are in normal region so it is considered as normal behaviour and is not reported.

\section{Experimental Results}

In this section, we evaluated the suggested framework using different experiments over resource constrained devices and personal computers. We performed two type of evaluations: first we used our own created dataset along with the ground truth to compute its accuracy and second we made time complexity analysis. The detailed analysis of our proposed framework is given in the subsequent sections.

\subsection{Alert transmission in IoT}

The control chart constructed for an interval is analyzed by checking the control limits of the graph. An interval is considered to be abnormal if more than two frames in it crosses the UCL boundary and drops from LCL range for both intensity and diversity over Y-, $\mathrm{X}$-axis, respectively. The current threshold of movement diversity for UCL is four and LCL is zero. Similarly, the threshold of movement intensity is 0.2 for LCL and six for UCL. The alert of abnormal breathing behavior is then transmitted in IoT received by the possible connected devices such smartphones, laptops, and smart display devices that can be viewed by the parents. A sample scenario for an alert transmission is shown in Fig. 4 where interval three will raise an alert in the IoT environment. 
Table I. Computer and RPi configuration details

\begin{tabular}{|c|c|c|}
\hline Unit & Computer system & RPi version 3, model B \\
\hline Operating system & $\begin{array}{c}\text { Windows 10 Pro } \\
\text { (64-bit) }\end{array}$ & Raspbian stretch \\
\hline Processor speed & @ 3.40GHz 3.40GHz & $1.2 \mathrm{GHz}$ \\
\hline Processor type & $\begin{array}{c}\text { Intel (R) } \\
\text { Core(TM) i7-3770 }\end{array}$ & ARM Cortex-A53 \\
\hline Installed RAM & 12GB & $1 \mathrm{~GB}$ \\
\hline GPU/Movidius & NVIDIA GTX 650 & N/A \\
\hline
\end{tabular}

\subsubsection{Experimental Settings}

The experiments are carried out over two types of machines, resource constrained device that is RPi and simple personal computer. The configuration of both these systems is given in Table I.

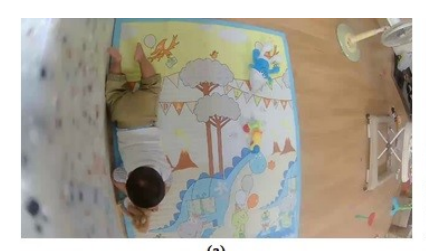

(a)

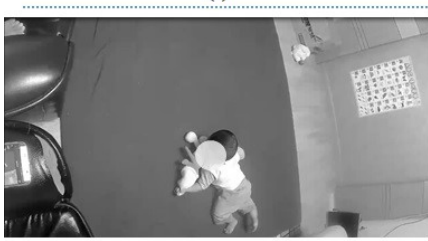

(d)

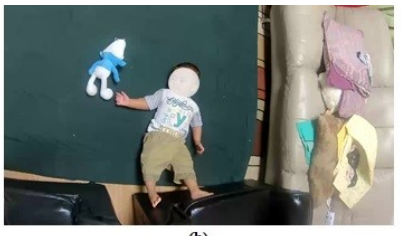

(b)

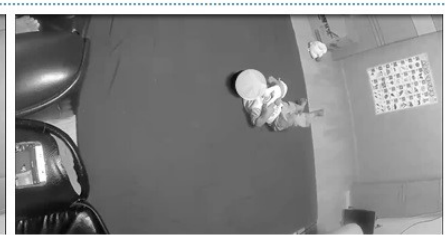

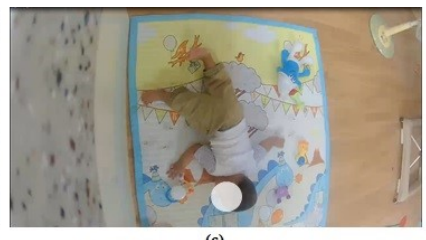

(c)

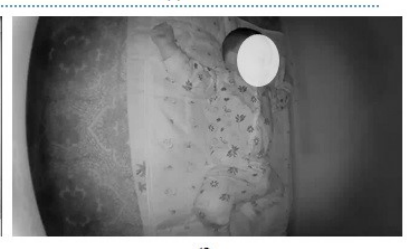

(f)

Figure 6. Sample frames from our own created dataset. Videos are recorded from baby while sleeping and playing. There are different heights, light scenarios, and angles of camera for recorded videos.

\subsubsection{Dataset Creation}

The domain of analyzing breathing behavior of baby is not enough mature so it lacks standard datasets. To overcome this issue, we made our own videos from a baby while playing and sleeping in both light and dark scenarios with different heights and angles. We used night vision camera to record videos of dark scenarios. Some sample frames from our own created dataset videos are shown in Fig. 6. In the overall dataset we have a total of 5 videos having length ranging from 2 to 4 minutes. Three videos are recorded in light conditions while two videos are captured at dark situation. The ground truth for status of the baby breathing behavior is put at corner of each video to make it simple enough for testing purposes. Videos of the dataset are encoded in H.264/AVC1. The evaluation of our proposed framework over this dataset is explained in subsequent section.

\subsubsection{Qualitative Analysis}

To evaluate the effectiveness of our proposed framework, we made a qualitative analysis. 
We made experiments with various parameters including video resolution, frame rate, response time for one interval. Furthermore, we evaluated our framework on both motion detection algorithms individually and also combine. Table II shows the parameters used in tables with their respective definitions and remarks. The evaluation metrics are precision, recall, and accuracy.

Table II. Description of parameters used in different tables

\begin{tabular}{|l|l|}
\hline \multicolumn{1}{|c|}{ Unit } & \multicolumn{1}{c|}{ Remarks } \\
\hline RT (sec) & $\begin{array}{l}\text { Response time (Execution time in seconds to process all the frames within } \\
\text { a single interval) }\end{array}$ \\
\hline Accuracy & Accuracy of the whole video for the specific FPS and resolution \\
\hline FPS & Frame per second \\
\hline RES & Resolution \\
\hline
\end{tabular}

\subsection{Precision}

Precision in our particular problem refers to the correctly predicted frames when compared with the available ground truth. As mentioned earlier we wrote ground truth of breathing behavior on the left corner of the input video. Thus we run our framework on those videos and checked for frame with various predictions. The possible outputs are: breathing frames marked as breathing, breathing as non-breathing, non-breathing as breathing, and non-breathing as non-breathing. So, the precision value for our task is the frames extracted correctly for breathing i.e., breathing as breathing divided by the sum of correctly and incorrectly predicted frames. The formula for precision value calculation is given in Eq. 1.

$$
\text { Precision }=\frac{\text { Breathing as breathing }}{(\text { Breathing as breathing+Breathing as non_breathing })}
$$

\subsection{Recall}

Recall value indicates ratio of relevant retrieved frames from the whole input video. Similar to precision value computation we divided the breathing as breathing predicted frames divided by the sum of miss predicted non-breathing as breathing frames and correctly predicted breathing frames. The formula to compute recall value for our system is given in Eq. 2.

$$
\text { Recall }=\frac{\text { Breathing as breathing }}{(\text { Breathing as breathing+Non_breathing as breathing })}
$$

\subsection{Accuracy}

Accuracy of our system for an input video is calculated by dividing the twice product of precision and recall on the sum of precision and recall. The formula for computing the accuracy is given in Eq. 3 .

$$
\text { Accuracy }=\frac{2 *(\text { Precision } * \text { Recall })}{(\text { Precision }+ \text { Recall })}
$$




\subsection{Personal/Smart computers}

The possible details about results of a sample video from our own created dataset is provided in Table III which shows accuracy and response time of frame difference, background subtraction, and hybrid method (frame difference and background subtraction) method. For experiments on computers we selected one interval as 0.5 seconds, thus there are different number of frames for each defined FPS in one interval. There are 15 frames per interval for 30 FPS, 7, 5, 3 and 2 frames per interval corresponding to 15, 10, 6 and 5 FPS, respectively.

Table III. Accuracy and response time of all the implemented methods for a personal computer

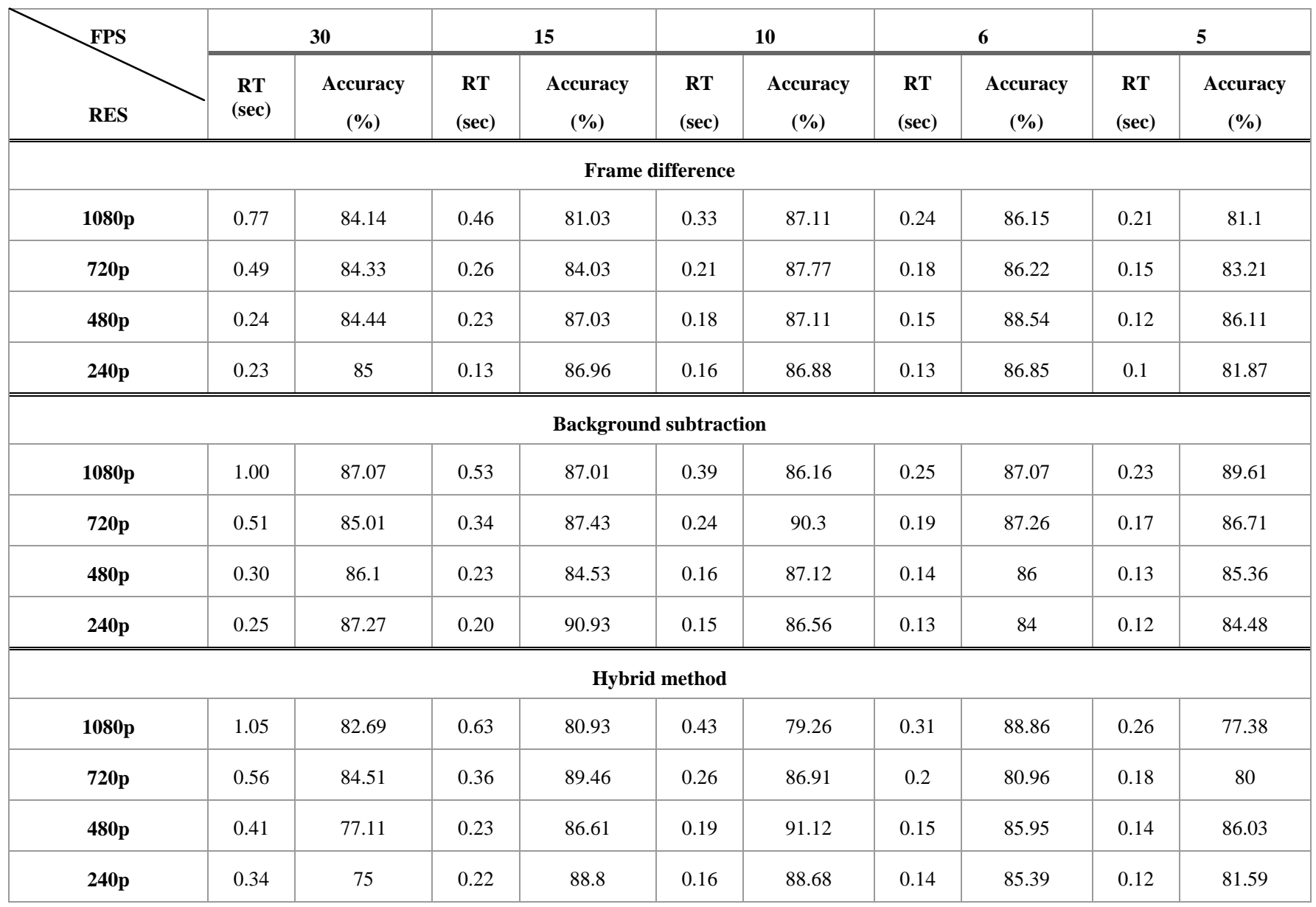

\subsection{Raspberry Pi}

The experiments performed on RPi are represented in Table IV. For experiments on RPi we considered a single interval as five frames and the response time written in all these tables refers to response time for a single interval. Description of the used parameters is given in Table II and the model of RPi and other necessary details are provided in Table I. 
Table IV. Accuracy and response time of all the implemented methods on RPi

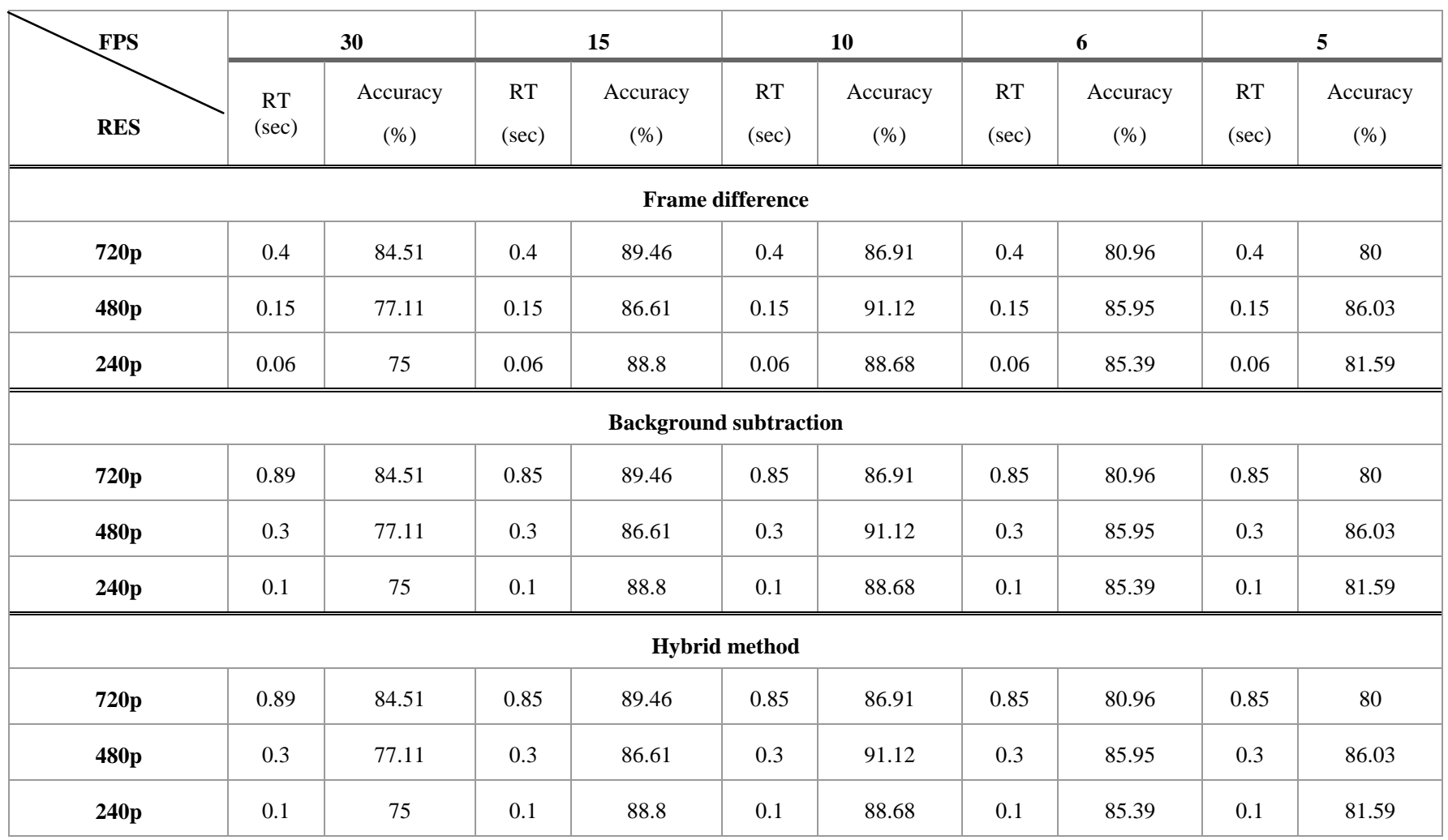

\section{Conclusions and Future Work}

In this article, we set a new trend in IoT by introducing a novel framework working in smart homes, healthcare centers, and nurseries to monitor and report the breathing status of the baby. Mainstream researchers while exploring different sensors working in an IoT ignore the usage of vision sensors for babies monitoring. Although there exist some traditional baby motion detection mechanisms relying only on breathing detection and have very limited applications and cannot be integrated with smart devices in IoT to make a complete system. Therefore, we present a framework functional in IoT environment for both resource constrained and non-constrained devices. The major steps of our framework include input acquisition from RPi camera, motion detection, control chart construction, behavior analysis, and alert transmission in wireless network. The input acquisition steps capture live baby video through a smart camera or RPi with an attached vision sensor and pass it to the motion detection module. The second steps acquire input frames and pass it through two algorithms to compute the motion inside them. Motion computed for a single interval having thresholded number of frames is represented through control chart. The UCL and LCL are checked and in case of crossing or falling from limits, an alert in the IoT is generated to inform the baby care takers or parents. The live baby streaming can also be viewed through smart devices to check the status of the baby. Time complexity and accuracy analysis of our proposed framework 
indicates the better performance of it in IoT environment for smart homes and nurseries.

The current framework has some limitations that need to be addressed in future. Motion detection mechanism we used is based on hand-crafted features where in future we intend to use some light weight CNN model for motion detection to effectively present actions/motion patterns [21]. Furthermore, we intend to test our methods on smartphones with various configurations to build a smart home with maximum utilization of all the IoT connected devices and make it easily available for doctors and baby care takers and smart hospitals.

\section{Acknowledgment}

This work was supported by the National Research Foundation of Korea (NRF) grant funded by the Korea government (MSIT) (No. 2019R1A2B5B01070067).

\section{Conflicts of Interest}

There is no conflict of interest.

\section{The link to GitHub code}

https://github.com/tanveer-hussain/Baby-behavior-monitoring-IoT

\section{References}

[1] S. Khan, K. Muhammad, S. Mumtaz, S. W. Baik, and V. H. C. de Albuquerque, "Energy-efficient deep CNN for smoke detection in foggy IoT environment," IEEE Internet of Things Journal, 2019.

[2] T. Hussain, K. Muhammad, J. Del Ser, S. W. Baik, and V. H. C. de Albuquerque, "Intelligent Embedded Vision for Summarization of Multi-View Videos in IIoT," IEEE Transactions on Industrial Informatics, 2019.

[3] A. Sun, W. Gong, R. Shea, and J. Liu, "A Castle of Glass: Leaky IoT Appliances in Modern Smart Homes," IEEE Wireless Communications, vol. 25, pp. 32-37, 2018.

[4] M. Mohammadi, A. Al-Fuqaha, M. Guizani, and J. Oh, "Semisupervised Deep Reinforcement Learning in Support of IoT and Smart City Services," IEEE Internet of Things Journal, vol. 5, pp. 624-635, 2018.

[5]M. Sajjad, M. Nasir, F. U. M. Ullah, K. Muhammad, A. K. Sangaiah, and S. W. Baik, "Raspberry Pi assisted facial expression recognition framework for smart security in law-enforcement services," Information Sciences, vol. 479, pp. 416-431, 2019.

[6] K. Muhammad, T. Hussain, and S. W. Baik, "Efficient CNN based summarization of surveillance videos for resource-constrained devices," Pattern Recognition Letters, 2018.

[7] M. W. Condry and C. B. Nelson, "Using Smart Edge IoT Devices for Safer, Rapid Response With Industry IoT Control Operations," Proceedings of the IEEE, vol. 104, pp. 938-946, 2016.

[8] K. Muhammad, R. Hamza, J. Ahmad, J. Lloret, H. Wang, and S. W. Baik, "Secure Surveillance Framework for IoT Systems Using Probabilistic Image Encryption," IEEE Transactions on Industrial Informatics, vol. 14, pp. 3679-3689, 2018.

[9] T. Hussain, K. Muhammad, A. Ullah, Z. Cao, S. W. Baik, and V. H. C. de Albuquerque, "Cloud-assisted multi-view video summarization using CNN and 
bi-directional LSTM," IEEE Transactions on Industrial Informatics, 2019.

[10] P. H. F. N. Sousa, N. M. M.; Almeida, J. S.; Rebouças Filho, P. P. and Albuquerque, V. H. C, "Intelligent Incipient Fault Detection in Wind Turbines based on Industrial IoT Environment," Journal of Artificial Intelligence and Systems, pp. 1-19, 2019.

[11] C. Taramasco, T. Rodenas, F. Martinez, P. Fuentes, R. Munoz, R. Olivares, et al., "A novel monitoring system for fall detection in older people," IEEE Access, vol. 6, pp. 43563-43574, 2018.

[12] C. Taramasco, T. Rodenas, F. Martinez, P. Fuentes, R. Munoz, R. Olivares, et al., "A novel low-cost sensor prototype for nocturia monitoring in older people," IEEE Access, vol. 6, pp. 52500-52509, 2018.

[13] S. Brangui, M. El Kihal, and Y. Salih-Alj, "An enhanced noise cancelling system for a comprehensive monitoring and control of baby environments," in Electrical and Information Technologies (ICEIT), 2015 International Conference on, 2015, pp. 404-409.

[14] M. P. Joshi and D. C. Mehetre, "IoT Based Smart Cradle System with an Android App for Baby Monitoring," in 2017 International Conference on Computing, Communication, Control and Automation (ICCUBEA), 2017, pp. 1-4.

[15] A. F. Symon, N. Hassan, H. Rashid, I. U. Ahmed, and S. T. Reza, "Design and development of a smart baby monitoring system based on Raspberry Pi and Pi camera," in Advances in Electrical Engineering (ICAEE), 2017 4th International Conference on, 2017, pp. 117-122.

[16] R. Palaskar, S. Pandey, A. Telang, A. Wagh, and R. Kagalkar, "An Automatic Monitoring and Swing the Baby Cradle for Infant Care," International Journal of Advanced Research in Computer and Communication Engineering, vol. 4, pp. 187-189, 2015.

[17] M. Koli, P. Ladge, B. Prasad, R. Boria, and P. N. J. Balur, "Intelligent Baby Incubator," in 2018 Second International Conference on Electronics, Communication and Aerospace Technology (ICECA), 2018, pp. 1036-1042.

[18]C. Pan, W. Zhao, S. Deng, W. Wei, Y. Zhang, and Y. Xu, "The Methods of Realizing Baby Crying Recognition and Intelligent Monitoring Based on DNN-GMM-HMM," in 2018 2nd IEEE Advanced Information Management, Communicates, Electronic and Automation Control Conference (IMCEC), 2018, pp. 352-356.

[19]Z. Zivkovic, "Improved adaptive Gaussian mixture model for background subtraction," in Pattern Recognition, 2004. ICPR 2004. Proceedings of the 17th International Conference on, 2004, pp. 28-31.

[20]Z. Zivkovic and F. Van Der Heijden, "Efficient adaptive density estimation per image pixel for the task of background subtraction," Pattern recognition letters, vol. 27, pp. 773-780, 2006.

[21] A. Ullah, K. Muhammad, J. Del Ser, S. W. Baik, and V. Albuquerque, "Activity recognition using temporal optical flow convolutional features and multi-layer LSTM," IEEE Transactions on Industrial Electronics, 2018. 